\title{
Post Operative Pleural Effusion and its PT Management- A Case Study
}

\section{Gurman Kaur ${ }^{1}$ and Satish Kumar Anumula ${ }^{2 *}$}

${ }^{1}$ BPT Student, Department of Physiotherapy, School of Physiotherapy and Paramedical Sciences, Lovely Professional University, Phagwara, Punjab, India

${ }^{2}$ Assistant Professor, Department of Physiotherapy, School of Physiotherapy and Paramedical Sciences, Lovely Professional University, Phagwara, Punjab, India

*Corresponding Author: Satish Kumar Anumula, Assistant Professor, Department of Physiotherapy, School of Physiotherapy and Paramedical Sciences, Lovely Professional University, Phagwara, Punjab, India.

DOI: 10.31080/ASOR.2020.03.0197
Received: April 17, 2020

Published: July 27, 2020

(C) All rights are reserved by Gurman Kaur and Satish Kumar Anumula.

\begin{abstract}
In patients with dilated cardiomyopathy and end stage heart failure heart transplantation is the definitive treatment strategy. Post-operative pulmonary complications are very common in any solid organ transplant surgery. In maximum of heart transplant surgeries pleural effusion is very common due to which the patient require prolong hospital stay and mechanical ventilation. This case study shows the severe right ventricular dysfunction who was underwent a heart transplant surgery. The past medical history reveals pulmonary embolism, hemicolectomy (cancer) and some risk factors including smoking. During the post-operative recovery period the patient received immunosuppressive treatment with prednisone, cyclosporine, mycophenolate. Their was reduced air entry in both lungs and the respiratory rate was $20 \mathrm{cpm}$. Physiotherapy goals for the patient is to improve cardiopulmonary function, prevent further cardiopulmonary complication and improve fatigability and cardiopulmonary endurance. The patient physiotherapy treatment for twice a day for minimum of 40 ins per session which included ambulation, limb mobilisation and respiratory exercises. This study concluded that positive airway pressure as a treatment approach was safe, feasible and easy during sub-intensive care setting while treating patients with post-operative pleural effusion.
\end{abstract}

Keywords: Pleural Effusion; Respiratory Exercises; Ambulation; Cardiomyopathy

\section{Introduction}

Dilated cardiomyopathy is characterized by the enlargement of systolic dysfunction and left ventricle. It presents with reduced cardiac output, congestion and heart failure [1]. The treatment available for dilated cardiomyopathy includes implantable cardiac defibrillators, pacemakers and pharmacological therapy [2]. Heart transplantation is the definitive treatment of choice for the patients with progressive dilated cardiomyopathy and end-stage heart failure refractory to medical or device therapy [2,3]. Organ transplantation leads to severe post-operative pulmonary complications and even can complicate or delay post-operative recovery $[4,5]$. Post-operative pulmonary complications following heart transplants found that $26 \%$ of 72 heart transplant recipients developed post-operative pleural effusion [4]. This can result in a longer stay in intensive care unit (ICU) along with prolong mechanical ventilation [4]. The treatment and early diagnosis of post-operative pulmonary complications include pleural effusion which contributes in reducing mortality and morbidity and following heart transplantation [6].

\section{Case Study}

A 54-year-old woman admitted in a hospital of Jalandhar, with a body mass index of 19.9, severe right ventricular dysfunction, normal coronary arteries and dilated cardiomyopathy undergone a heart transplant surgery. Moreover, episodes of multiple ventricular arrhythmias were recorded and treated with an implantable cardioverter defibrillator (ICD). The patient received the transplant surgery without any complications and ICD was removed. At the end of the surgical procedure, drains were placed (pleural, pericardial and retrosternal). During the post-operative recovery period, the patient received standard immunosuppressive treatment with prednisone, cyclosporine, and mycophenolate mofetil.

\section{Past history}

The clinical history included hemicolectomy (cancer), previous pulmonary embolism and moderate risk factors including smoking. Before the heart transplant, the patient underwent repeated infusions of inotropic agents and no exercise testing was performed. 
Examination

- $\quad$ CNS

- Patient is conscious, alert and well oriented.

- $\quad$ CHEST

- Vesicular breath sounds

- $\quad$ Reduced air entry in both lungs' zones more in the left middle. field.

- $\quad$ RR-20 cpm

- $\quad$ CTTD tube in-situ draining serous fluid.

- $\quad$ CVS

- $\quad$ B.P- $145 / 90 \mathrm{mmHg}$

- Pulse-101/min.

- $\quad$ MMT

- Gross muscle power is $4+$ in the upper limb

- Gross muscle power is+ in the lower limb.

Physiotherapy goals

a) To improve cardiopulmonary function

b) To prevent further cardiopulmonary complication

c) To improve fatigability and cardiopulmonary endurance.

\section{Physiotherapy management}

On the postoperative day (POD) 3the patient was discharged from the ICU and the patient was then transferred to the cardiac ward where her vital and hemodynamics were checked and found to be stable and her respiratory dynamics were unchanged. There were no bronchial secretions present. While the patient was in the ICU Physiotherapy was initiated with active and passive range of motion exercises of both upper limbs and lower limbs along with respiratory training (deep breathing exercises and assisted cough) [7]. Starting from the day of admission to the sub-intensive care ward, physiotherapy comprising respiratory and motor exercises twice daily (40 minutes a session) continued from POD 5 to 10 . These exercises consisted of:

- Initially range of motion exercises were carried out in supine position which involves assisted mobilization of upper limb and lower limb [8].

- Postural passages from (supine to sitting), in which the patient was assisted and encouraged to gain a sitting position at the edge of bed. Active antigravity exercises were carried out while sitting to enhance trunk stability (leg extension, arm elevation and maintaining a sitting position).

- $\quad$ Deep breathing exercises in both supine and sitting positions ( 2 - 3 sets of 10 repetitions twice daily) [9]. The patient was instructed to inhale deeply and then hold for 2 - 4 seconds to maintain maximum inspiration before expiring [9].
- In supine position, controlled breathing techniques were taught using upper and lower chest with relaxed shoulders [9]. The patient was instructed to put her hand on upper abdomen to feel the rise of abdomen during inspiration and descend during expiration.

DDespite of medical treatment (furosemide $80 \mathrm{mg} /$ day) and volume status (mean atrial pressure effusion with bibasilar dysventilation was diagnosed using imaging techniques. Chest radiographs (Figure 1) and a thoracic computed tomography scan (Figure 2) suggested an approximately $350 \mathrm{~mL}$ effusion. Due to these findings, respiratory treatment was started to counteract the deteriorating the condition of the patient. In addition to the described physiotherapy, the EzPAP system was added as a positive pressure device four times daily (twice each morning and afternoon) from POD 8 to 12 [10]. In our case, the device was POD 8 to 12. In our

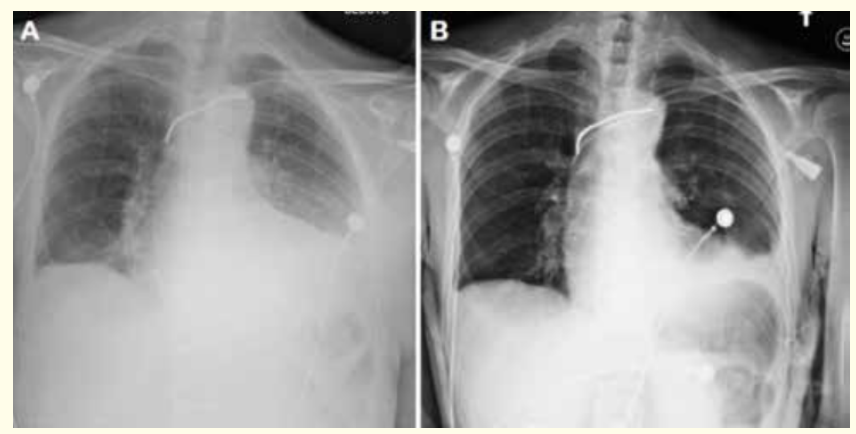

Figure 1: On post-operative day 7, a pleural effusion was evident in the left lung with the ascent of the diaphragm; (b) On post-operative day 12 chest $x$-ray examination showed the reduction of the inhibitory phenomena.

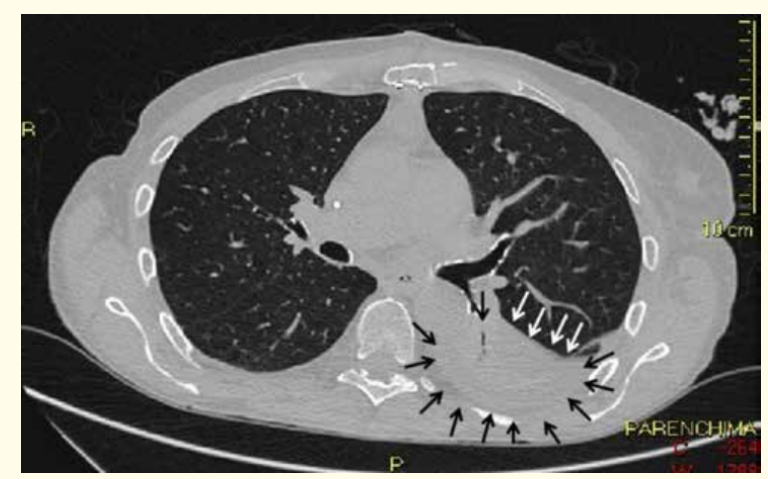

Figure 2: Left hypodense pleural effusion with atelectasis of the lower left lobe (arrowed area). 
case, the device was used with a mouthpiece and the exercises were performed in a sitting position. At the end of each exercise session, the mouthpiece was cleaned, disinfected, and placed in a bag to prevent contamination. Each session lasted about 15 minutes. Respiratory therapy (deep breathing exercises and incentive spirometry) was continued until the discharge on POD 26.
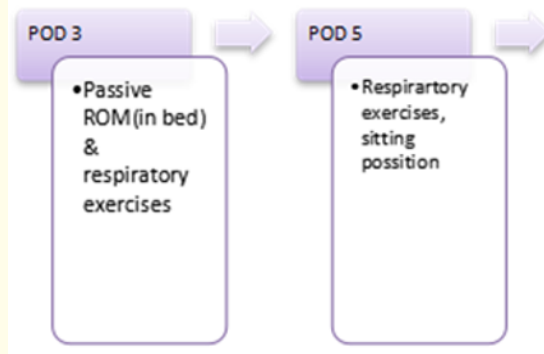

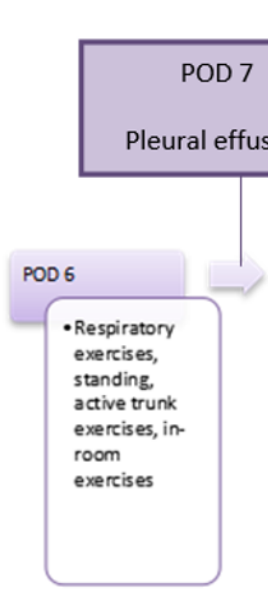

Figure 3
The pressure was set to $8 \mathrm{~cm} \bullet \mathrm{H} 2 \mathrm{O}$ on POD 8 , and it was increased to $12 \mathrm{~cm} \bullet \mathrm{H} 20$ on POD 9 and then $15 \mathrm{~cm} \bullet \mathrm{H} 20$ on POD 10 - 12. Diuretic therapy with intravenous furosemide had been started in the first post-operative days at $240 \mathrm{mg}$ /day and then reduced to $80 \mathrm{mg} /$ day; the oral furosemide was increased to 200 $\mathrm{mg} /$ day on POD 8 and $250 \mathrm{mg}$ /day on POD 9 to 11 .

\section{Discussion}

In this case study, the worsening pulmonary condition could have led to infective complications and simultaneously necessitated a more invasive therapeutic approach, i.e. thoracentesis [11]. Haemodynamic and clinical stability allows a noninvasive therapeutic option comprising of pharmacological therapy with diuretics, application of positive pressure using EzPAP, postural transfers, ambulation, range of motion exercises and respiratory exercises. To our knowledge, this is the first reported use of the EzPAP in heart transplant patients. Pleural effusion causes chest wall expansion and decrease lung volume. For which pulmonary function test is a main outcome measure used [12]. The main goals of physiotherapy treatment in patients with respiratory pathologies are to decrease the risk associated with bed rest and intensive care and to increase the functional capacity of the patient [13]. Medical treatment when added to the physiotherapy treatment results in decreased hospital stay of patients, improves chest radiographs and parameters of spirometer in the patients with pleural effusion [11]. The aim of physiotherapy treatment is to apply cost-effective and advance therapeutic modalities which improves residual func- tion, makes patient independent and enhance the quality of life [14].

\section{Conclusion}

The study has integrated therapeutic model which include medications and exercises which are effective during the worsening conditions of pleural effusion after the surgery. In this case study, positive airway pressure as a treatment approach was safe, feasible and easy during sub-intensive care setting while treating patients with post-operative pleural effusion.

\section{Bibliography}

1. NK Lakdawala., et al. "Dilated Cardiomyopathy". Circulation: Arrhythmia and Electrophysiology 6.1 (2013): 228-237.

2. Hershberger RE and A Morales. "Dilated cardiomyopathy: overview". University of Washington (2007).

3. EP Alraies MC. "Adult heart transplant: indications and outcomes". Journal of Thoracic Disease 6.8 (2014): 1120-1128.

4. KO Camkiran Firat A. "Early postoperative pulmonary complications after heart transplantation". Transplantation Proceedings 47.4 (2015): 1214-1216.

5. De Gasperi A., et al. "Pulmonary complications in patients receiving a solid-organ transplant". Current Opinion in Critical Care 20.4 (2014): 411-419. 
6. JJ Gagnier., et al. "The CARE guidelines: Consensus-based clinical case report guideline development". Journal of Clinical Epidemiology 67.1 (2014): 46-51.

7. S Berney., et al. "Physiotherapy in Critical Care in Australia". Cardiopulmonary Physical Therapy Journal 23.1 (2012): 1925.

8. M Green., et al. "Mobilization of intensive care patients: A multidisciplinary practical guide for clinicians". Journal of Multidisciplinary Healthcare 9 (2016): 247-256.

9. MH Jang., et al. "Pulmonary and physical rehabilitation in critically ill patients”. Acute and Critical Care 34.1 (2019): 1-13.

10. A D Rieg., et al. "[EzPAP ${ }^{\circ}$ therapy of postoperative hypoxemia in the recovery room: experiences with the new compact system of end-expiratory positive airway pressure]". Anaesthesist 61.10 (2012): 867-874.

11. G Valenza-Demet., et al. "The effects of a physiotherapy programme on patients with a pleural effusion: a randomized controlled trial". Clinical Rehabilitation 28.11 (2014): 10871095.

12. Massimiliano Polastri CS. "Post-operative pleural effusion in a heart transplant recipient: A single case study of physiotherapy treatment". International Journal of Therapy and Rehabilitation 24.7 (2017): 302-305.

13. R Topp., et al. "The effect of bed rest and potential of prehabilitation on patients in the intensive care unit". AACN Clinical Issues 13.2 (2002): 263-276.

14. K Stiller. "Physiotherapy in intensive care: Towards an evidence-based practice". Chest 118.6 (2000): 1801-1813.

\section{Assets from publication with us}

- Prompt Acknowledgement after receiving the article

- Thorough Double blinded peer review

- Rapid Publication

- Issue of Publication Certificate

- High visibility of your Published work

Website: https://www.actascientific.com/

Submit Article: https://www.actascientific.com/submission.php Email us: editor@actascientific.com

Contact us: +919182824667 